\title{
Secure elliptic curves and their performance
}

\author{
V. Gayoso Martínez ${ }^{1}$, L. Hernández Encinas ${ }^{1}$, \\ A. Martín Muñoz ${ }^{1}$, and R. Durán Díaz ${ }^{2}$ \\ 1 Institute of Physical and Information Technologies (ITEFI) \\ Spanish National Research Council (CSIC), Madrid, Spain \\ \{victor.gayoso, luis, agustin\}@iec.csic.es \\ 2 Department of Automatics, University of Alcalá, Alcalá de Henares, Spain \\ raul.duran@uah.es
}

\begin{abstract}
Elliptic Curve Cryptography (ECC) is a branch of publickey cryptography based on the arithmetic of elliptic curves. In the short life of ECC, most standards have proposed curves defined over prime finite fields satisfying the curve equation in the short Weierstrass form. However, some researchers have started to propose as a securer alternative the use of Edwards and Montgomery elliptic curves, which could have an impact in current ECC deployments. This contribution evaluates the performance of the three types of elliptic curves using some of the examples provided by the initiative SafeCurves and a Java implementation developed by the authors, which allows us to offer some conclusions about this topic.
\end{abstract}

Keywords: Edwards curves, Elliptic Curve Cryptography, Java, Montgomery curves, point arithmetic, Weierstrass curves

\section{Introduction}

In 1987, Neal Koblitz [1] and Victor Miller [2] independently suggested using elliptic curves defined over finite fields for implementing different cryptosystems. This branch of public-key cryptography is typically known as ECC (Elliptic Curve Cryptography), and its security is based on the difficulty of solving the ECDLP (Elliptic Curve Discrete Logarithm Problem), a mathematical problem which is at least as difficult to solve as the IFP (Integer Factorization Problem) used in the RSA algorithm or the DLP (Discrete Logarithm Problem) employed by the ElGamal algorithm.

One of the most important aspects when working with elliptic curves is their selection mechanism. Even though some standards include several sample curves or even the description of the procedure for generating them (for example, X9.63 [3], IEEE 1363 [4] or NIST FIPS 186-4 [5]), in most cases the information contained in those standards has important limitations, such as the lack of clarity in the selection procedure regarding the seeds and prime numbers involved or the insufficient explanation for some of the requirements taken into account. 
In this scenario, at the beginning of the last decade a working group called ECC Brainpool focused on this topic and elaborated a first set of recommendations in 2005 [6]. Five years later, the Brainpool specification was revised and published as an RFC (Request for Comments) [7]. The Brainpool initiative is usually considered as the first international effort with the goal of producing a truly transparent curve generation procedure. The curves suggested in its specification, described using the short Weierstrass form, were initially considered by many experts to be secure without any hint of doubt.

Some time after that, Bernstein and Lange [8] published an analysis in which they reviewed the existing elliptic curve generation mechanisms, including the one devised by Brainpool. The main result of their study was that all the schemes included in the standards overlooked some aspects of the ECDLP security and, for that reason, required to increase the complexity of the implementations in such a way that it opened the door to side channel attacks [8]. As a solution, Bernstein and Lange decided to propose new curves different to those provided by previous specifications. Going one step further, they evaluated 20 curves of different types obtained from several sources, showing that the only curves that were able to fulfil all their security requirements were the Edwards and Montgomery curves.

As another example of the concern about the reliability of the curve generation process, in 2016 Baignères [9] produced a new Edwards elliptic curve (called the million dollar curve for reasons that will be explained later) using a technique that put the emphasis on the randomness of the data used in the procedure [9].

However, from the point of view of availability, both Montgomery and Edwards curves have not been popular choices so far, and in that respect traditional curves in the short Weierstrass form are the dominant options both in hardware and software implementations. In addition to that, the extra security offered by Edwards and Montgomery curves could affect the performance of the point operations which are the core of scalar multiplications (the product of a point of the elliptic curve by an integer, an operation needed in any protocol involving elliptic curves).

Based on the work by Bernstein and Lange, this contribution (which extends the work presented in [10]) addresses the issue of the performance of the Edwards and Montgomery curves proposed at SafeCurves compared to Weierstrass curves. In order to do that, Section 2 provides a brief review of the main concepts regarding generic elliptic curves and, most specifically, of those defined using the Weierstrass, Edwards, and Montgomery forms. Section 3 includes actual examples available for developers of real-world elliptic curves of the three types. Section 4 contains the algorithms that minimise the number of finite field operations in the point addition and doubling procedures, which allows us to compare their theoretic complexity to the practical results obtained after implementing those operations in a Java application. Section 5 shows the performance results obtained with the aforementioned implementation. Finally, in Section 6 we give the interested readers our conclusions on this topic. 


\section{Elliptic curves}

\subsection{Definition}

An elliptic curve defined over a field $\mathbb{F}$ is a cubic, non-singular curve whose points $(x, y) \in \mathbb{F} \times \mathbb{F}$ verify the following equation, known as the Weierstrass equation:

$$
E: y^{2}+a_{1} x y+a_{3} y=x^{3}+a_{2} x^{2}+a_{4} x+a_{6},
$$

where $a_{1}, a_{2}, a_{3}, a_{4}, a_{6} \in \mathbb{F}$ and $\Delta \neq 0$, where $\Delta$ is the discriminant of $E$ that can be computed as follows [11]:

$$
\begin{aligned}
& \Delta=-d_{2}^{2} d_{8}-8 d_{4}^{3}-27 d_{6}^{2}+9 d_{2} d_{4} d_{6}, \\
& d_{2}=a_{1}^{2}+4 a_{2}, \\
& d_{4}=2 a_{4}+a_{1} a_{3}, \\
& d_{6}=a_{3}^{2}+4 a_{6}, \\
& d_{8}=a_{1}^{2} a_{6}+4 a_{2} a_{6}-a_{1} a_{3} a_{4}+a_{2} a_{3}^{2}-a_{4}^{2} .
\end{aligned}
$$

An elliptic curve point is singular if and only if the partial derivatives of the curve equation are null at that point. The curve is said to be singular if it possesses at least a singular point, while it is non-singular if it does not have any such points. A singular curve defined over the field $\mathbb{R}$ can be easily identified by the presence of cusps or self-intersections. Only non-singular curves are of interest in Cryptography.

The non-homogeneous Weierstrass equation can also be expressed in the following homogeneous form [12]:

$$
Y^{2} Z+a_{1} X Y Z+a_{3} Y Z^{2}=X^{3}+a_{2} X^{2} Z+a_{4} X Z^{2}+a_{6} Z^{3},
$$

where once again $a_{1}, a_{2}, a_{3}, a_{4}, a_{6} \in \mathbb{F}$. This equation defines a curve which includes a special point called the point at infinity, which is typically represented as $\mathcal{O}=[0: 1: 0]$ and that has no correspondence with any point of the nonhomogeneous form. However, this point is very important as it works as the identity element of the addition operation when working with Weierstrass and Montgomery elliptic curves.

\subsection{Elliptic curves over finite fields}

Most cryptosystems defined over elliptic curves use only one of the following finite fields $\mathbb{F}_{q}$ with $q=p^{m}$ elements: prime fields $\mathbb{F}_{p}$ (where $p$ is an odd prime number and $m=1$ ) and binary fields $\mathbb{F}_{2^{m}}$ (where $m$ can be any positive integer). However, due to a combination of licence issues and security concerns [13], prime fields have been favoured in the latest specifications at the expense of binary fields (see, for example, Brainpool [7], NSA Suite B [14] or BSI TR-03111 [15]). Following that criterion, in what follows we have accordingly focused our study on prime fields. 
The peculiarities of prime fields allow to simplify the general Weierstrass equation and to obtain in the process what is called the short Weierstrass form represented as $y^{2}=x^{3}+a x+b$, where $4 a^{3}+27 b^{2} \not \equiv 0(\bmod p)$.

As in the case of the general Weierstrass equation, the identity element of the short Weierstrass form is the point at infinity $\mathcal{O}$, while the opposite element of a point $P=(x, y)$ is the point $-P=(x,-y)$. Adding two points $P_{1}=\left(x_{1}, y_{1}\right)$ and $P_{2}=\left(x_{2}, y_{2}\right)$ such that $P_{1} \neq \pm P_{2}$ produces a point $P_{3}=\left(x_{3}, y_{3}\right)$ whose coordinates can be computed as follows [16]:

$$
\begin{gathered}
x_{3}=\frac{\left(y_{2}-y_{1}\right)^{2}}{\left(x_{2}-x_{1}\right)^{2}}-x_{1}-x_{2}, \\
y_{3}=\frac{\left(2 x_{1}+x_{2}\right)\left(y_{2}-y_{1}\right)}{x_{2}-x_{1}}-\frac{\left(y_{2}-y_{1}\right)^{3}}{\left(x_{2}-x_{1}\right)^{3}}-y_{1} .
\end{gathered}
$$

In comparison, when $P_{1}=P_{2}$ it is necessary to use an alternative addition formula, so in this case the point $P_{3}=2 P_{1}$ obtained through the doubling operation has the following coordinates [16]:

$$
\begin{gathered}
x_{3}=\frac{\left(3 x_{1}^{2}+a\right)^{2}}{\left(2 y_{1}\right)^{2}}-2 x_{1}, \\
y_{3}=\frac{\left(3 x_{1}\right)\left(3 x_{1}^{2}+a\right)}{2 y_{1}}-\frac{\left(3 x_{1}^{2}+a\right)^{3}}{\left(2 y_{1}\right)^{3}}-y_{1} .
\end{gathered}
$$

Edwards curves were introduced in [17] and are defined according to the equation $x^{2}+y^{2}=c^{2}\left(1+d x^{2} y^{2}\right)$, where $c d\left(1-d c^{4}\right) \not \equiv 0(\bmod p)$. An Edwards curve is said to be complete if $d$ is not a quadratic residue module $p$.

The identity element in Edwards curves is the point $(0, c)$ while, taking into consideration a point $P=(x, y)$, its opposite element is $-P=(-x, y)$. The result of adding any two points $P_{1}=\left(x_{1}, y_{1}\right)$ and $P_{2}=\left(x_{2}, y_{2}\right)$ is the point $P_{3}=\left(x_{3}, y_{3}\right)$ whose coordinates can be computed as follows [16]:

$$
\begin{aligned}
& x_{3}=\frac{x_{1} y_{2}+y_{1} x_{2}}{c\left(1+d x_{1} x_{2} y_{1} y_{2}\right)}, \\
& y_{3}=\frac{y_{1} y_{2}-x_{1} x_{2}}{c\left(1-d x_{1} x_{2} y_{1} y_{2}\right)} .
\end{aligned}
$$

It should be noted that, in the case of Edwards curves, the equation for both adding two points $P_{1}$ and $P_{2}$ such that $P_{1} \neq \pm P_{2}$ and doubling a point are exactly the same. Moreover, it is not necessary to implement any logic for detecting if the points to be added are such that $P_{2}=-P_{1}$, as the Edwards addition equations also take into account that circumstance.

Finally, Montgomery curves conform to the equation $B y^{2}=x^{3}+A x^{2}+x$, where $B\left(A^{2}-4\right) \not \equiv 0(\bmod p)$. As in the case of Weierstrass curves, the identity element in Montgomery curves is the point at infinity $\mathcal{O}$, while the opposite element of $P=(x, y)$ is the point $-P=(x,-y)$. The addition of two points 
$P_{1}=\left(x_{1}, y_{1}\right)$ and $P_{2}=\left(x_{2}, y_{2}\right)$ such that $P_{1} \neq \pm P_{2}$ is the point $P_{3}=\left(x_{3}, y_{3}\right)$ with the following coordinates [16]:

$$
\begin{gathered}
x_{3}=\frac{B\left(y_{2}-y_{1}\right)^{2}}{\left(x_{2}-x_{1}\right)^{2}}-A-x_{1}-x_{2}, \\
y_{3}=\frac{\left(2 x_{1}+x_{2}+A\right)\left(y_{2}-y_{1}\right)}{x_{2}-x_{1}}-\frac{B\left(y_{2}-y_{1}\right)^{3}}{\left(x_{2}-x_{1}\right)^{3}}-y_{1} .
\end{gathered}
$$

Unlike Edwards curves, it is necessary to use different equations for the doubling operation, so in this case the coordinates of the point $P_{3}=2 P_{1}$ can be computed as follows [16]:

$$
\begin{gathered}
x_{3}=\frac{B\left(3 x_{1}^{2}+2 a x_{1}+1\right)^{2}}{\left(2 B y_{1}\right)^{2}}-A-2 x_{1}, \\
y_{3}=\frac{\left(3 x_{1}+A\right)\left(3 x_{1}^{2}+2 A x_{1}+1\right)}{2 B y_{1}}-\frac{B\left(3 x_{1}^{2}+2 A x_{1}+1\right)^{3}}{\left(2 B y_{1}\right)^{3}}-y_{1} .
\end{gathered}
$$

\subsection{Transforming formulas}

While Edwards curves can be always expressed in the Montgomery and short Weierstrass forms, the converse is not always possible. In order to transform an Edwards elliptic curve into a Montgomery elliptic curve as displayed in $\S 2.2$, it is necessary to use the following equivalence formulas [8]:

$$
A=B-2, \quad B=\frac{4}{1-d c^{4}} .
$$

In this way, a curve point $\left(x_{E}, y_{E}\right)$ which belongs to an Edwards curve can be converted to a point $\left(x_{M}, y_{M}\right)$ of the associated Montgomery curve, where the equations for obtaining $\left(x_{M}, y_{M}\right)$ are as follows:

$$
x_{M}=\frac{c+y_{E}}{c-y_{E}}, \quad y_{M}=c \frac{x_{M}}{x_{E}} .
$$

Besides, in order to transform a Montgomery elliptic curve into the short Weierstrass form as represented in $\S 2.2$, it is necessary to use the following equivalences [8]:

$$
a=\frac{3-A^{2}}{3 B^{2}}, \quad b=\frac{2 A^{3}-9 A}{27 B^{3}}
$$

In this specific case, a curve point $\left(x_{M}, y_{M}\right)$ belonging to a Montgomery curve can be converted to a point $\left(x_{W}, y_{W}\right)$ of the associated short Weierstrass curve, where the transforming equations are the following ones:

$$
x_{W}=\frac{x_{M}+\frac{A}{3}}{B}, \quad y_{W}=\frac{y_{M}}{B} .
$$




\section{Public proposals using different types of curves}

The three types of elliptic curves described in the previous section (Weierstrass, Edwards, and Montgomery) are not useful if they cannot be used in real-life deployments. In this section, three examples of elliptic curve generation procedures using different types of curves are described.

\subsection{Brainpool}

At the beginning of the new millennium, a European consortium of companies and government agencies led by the Bundesamt für Sicherheit in der Informationstechnik (BSI) was formed in order to study the topic of secure elliptic curve generation. The group was named ECC Brainpool (henceforth simply Brainpool) and, apart from the BSI, some of the most relevant companies and public institutions that took part in the effort were G\&D, Infineon Technologies, Philips Electronics, Gemplus (now part of Gemalto), Siemens, the Technical University of Darmstadt, T-Systems, Sagem Orga, and the Graz University of Technology.

In 2005, Brainpool delivered the first version of a document entitled "ECC Brainpool standard curves and curve generation" [6], which was eventually published as an RFC memorandum in 2010, the "Elliptic Curve Cryptography (ECC) Brainpool standard curves and curve generation" [7]. The following paragraphs present the main characteristics of the Brainpool procedure.

Key length. The Brainpool procedure only manages elliptic curves defined over prime fields expressed in the short Weierstrass form. The key lengths allowed by Brainpool are 160, 192, 224, 256, 320, 384, and 512 bits [7].

Seed generation. The seeds used in Brainpool are generated in a systematic and comprehensive way. The seeds for the parameter $p$ are obtained as the first 7 substrings of 160 bits each of the number $\pi \cdot 2^{1120}=$ Seed_p_160 || Seed_p_192|| . . $\|$ ||Seed_p_512 ||Remainder, where || denotes the concatenation operator and the term Seed_p_n refers to the 160 bits used as a seed in the curve of bitlength $n$ [7]. Besides, seeds for the curve parameters $a$ and $b$ are obtained as the first 7 substrings of 160 bits each of the number $\left\lfloor e \cdot 2^{1120}\right\rfloor=$ Seed_ab_160 ||Seed_ab_192|| . . $\|$ ||Seed_ab_512||Remainder.

Seed to candidate conversion. Brainpool uses SHA-1 [7] during the process of finding candidates for the fixed-length parameters $p, a$, and $b$. Although SHA-1 is not recommended as a hashing function in security environments (e.g. digital signatures), it is important to notice that in this context it is only used for generating candidate values.

As the output of the hashing function SHA-1 is 160 bits, and for different curves the length of the resulting parameters must be necessarily different, Brainpool performs a loop concatenating several SHA-1 outputs until the concatenated number has the proper bit length [7]. 
In addition to that, Brainpool uses two functions to generate the candidates, one for $p$ and another one for $a$ and $b$. Those functions are very similar, in fact the only difference is that the most significant bit of $a$ and $b$ is forced to be 0 . Given that another requirement states that the most significant bit of $p$ must be 1 , this implies that the parameters $a$ and $b$ generated are such that $a, b<p[7]$.

Cofactors. In order to generate cryptographically strong elliptic curves, it is necessary to compute the number of points of the elliptic curve and to determine if that value is a prime number or if it has a small cofactor. In this regard, the Brainpool specifications only accept curves whose number of points is a prime number [7]. This means that Brainpool curves cannot be transformed into the twisted Edwards or Montgomery forms, as in those types of curves the number of points is always divisible by 4 .

\subsection{SafeCurves}

Bernstein and Lange explain in their website SafeCurves [8] how all the standards that include recommended elliptic curves have addressed ECDLP security but not what they refer to as ECC security.

Bernstein and Lange state that elliptic curves designed to be ECDLP secure may be attacked if they are not implemented properly, which would allow, for example, to produce incorrect results for some rare curve points, leak secret data when the input is not a curve point, provide secret data through branch timing, or leak secret data through cache timing. The authors believe that secure implementation of the previously mentioned standard curves is theoretically possible but very difficult in practice. In order to avoid those potential implementation problems, the authors propose to use new curves which allow simple, secure implementations.

SafeCurves includes an evaluation of 20 curves taken from several sources (one SEC 2 curve, one ANSSI curve, two curves provided as examples of bad design, two Brainpool curves, three NIST curves, five Montgomery curves, and six Edwards curves) showing that some of them do not pass all their security requirements, which are divided into three main groups, as described in the next paragraphs: curve parameters, ECDLP security, and ECC security.

Curve parameters. Following other standards and initiatives, SafeCurves only uses elliptic curves defined over prime finite fields.

Regarding the cofactor, while in all the Brainpool curves its value is always 1 (i.e., the order of the generator $G$ equals the number of points of the curve), the Montgomery and Edwards examples from SafeCurves have cofactors whose value is either 4 or 8 . According to Bernstein and Lange, small subgroups attacks, which could take advantage of curves with a cofactor greater than 1 , can be easily deactivated either by implementing the appropriate software checks or by the inherent characteristics of the Montgomery and Edwards curves described in [8].

As for the key length, the curves analysed by SafeCurves have one of the following lengths: 221, 222, 251, 255, 382, 383, 414, 448, 511, and 521 bits. 
ECDLP security. Bernstein and Lange analysed how resistant the curves are against some well-known attacks to the ECDLP. More specifically, they studied the resistance against the Pollard $\rho$ method, the MOV attack, and the Weil descent attacks.

In addition to that, SafeCurves checks the degree to which the elliptic curve generation process is explained (e.g., the choice of the seed values, the operations performed on them to derive the parameters, etc.).

ECC security. The authors consider that the most important computation in ECC is the single-scalar multiplication. SafeCurves requires curves to support simple and constant-time multiplications, avoiding conflicts between efficiency and security.

Some of the aspects analysed in this section of their study are the security and efficiency of the scalar multiplication operations, the resistance to small subgroup attacks combined with the usage of incorrect curve parameters, the resistance to side channel attacks using the bifurcations of the logic implementing scalar multiplications and point additions, and the difficulty for an external observer to distinguish between the numeric representation of an elliptic curve point and a random binary string.

Using the previously described criteria, the authors of SafeCurves evaluated the aforementioned 20 curves. As it was expected given the content of the requirements, all the curves originally described using the short Weierstrass form do not satisfy at least three of the four ECC security requirements. Apart from that, the SEC 2 curve does not pass the complex-multiplication field discriminant test, while the NIST and the ANSSI curves are considered to be manipulable. Regarding the two Brainpool curves considered in the comparison, they pass all the parameter and ECDLP security requirements, but they fail to pass some of the ECC security requirements.

\subsection{Million dollar curve}

Another alternative proposal is the one known as the Million Dollar Curve [18]. This curve tries to avoid the problem of the lack of reliability in the choice of the parameters by using what the authors call publicly verifiable RNGs (Random Number Generators). The method extracts a seed that is used in the BlumBlum-Shub bit generator [19]. After that, it uses the binary string produced by this generator as input for a filtering function that deterministically identifies secure and uniformly distributed parameters from uniform bit strings.

The publicly verifiable randomness is provided by a collection of data associated to a list of national lotteries around the world elaborated in February 2016, which anyone interested can verify. The Million Dollar Curve, denoted either as MDC201601 or MDCurve201601 (Million Dollar Curve of January 2016) is a 256-bit Edwards curve defined over a prime finite field $\mathbb{F}_{p}$ with cofactor 4 that can be used as an alternative to Brainpool's P-256 curve or SafeCurve's Curve25519 [9]. 


\section{Implementation}

As it is well known, there are two possible approaches when implementing mathematics using modern programming languages: either minimise the amount of memory used by the implementation, or minimise the number of operations. Given that the goal of this contribution is to compare the performance of the different curve implementations, the approach taken has been to reduce as much as possible the number of finite field operations, which can be of the following types: addition/subtraction, multiplication, squaring, and multiplicative inversion (for the sake of simplicity, in what follows we will refer to both additions and subtractions simply as additions).

Depending on the processor, operating system, and programming language, the exact relationship between the computing cost of those operations may vary, and for instance in [16] there are several examples with different equivalences between the inversion, multiplication and squaring costs, though it is commonly accepted that the addition cost is negligible compared to that of the other field operations.

As our implementation was designed to work on personal computers, the amount of memory used was not a concern; however, if the goal had been to implement the curve arithmetic in smart cards, it would have been necessary to adopt the opposite approach, as in that case the amount of memory for card applications is certainly limited and reusing variables would have been an unavoidable need.

Algorithm 1 shows the details of the point addition and point doubling operations in Weierstrass curves. In the case of point addition, the elements involved are $P_{1}=\left(x_{1}, y_{1}\right)$ and $P_{2}=\left(x_{2}, y_{2}\right)$ such that $P_{1} \neq \pm P_{2}$. This point addition implementation needs 6 additions, 2 multiplications, 1 squaring, and 1 inversion in $\mathbb{F}_{p}$, while the point doubling implementation requires 8 additions, 2 multiplications, 2 squarings, and 1 inversion in $\mathbb{F}_{p}$.

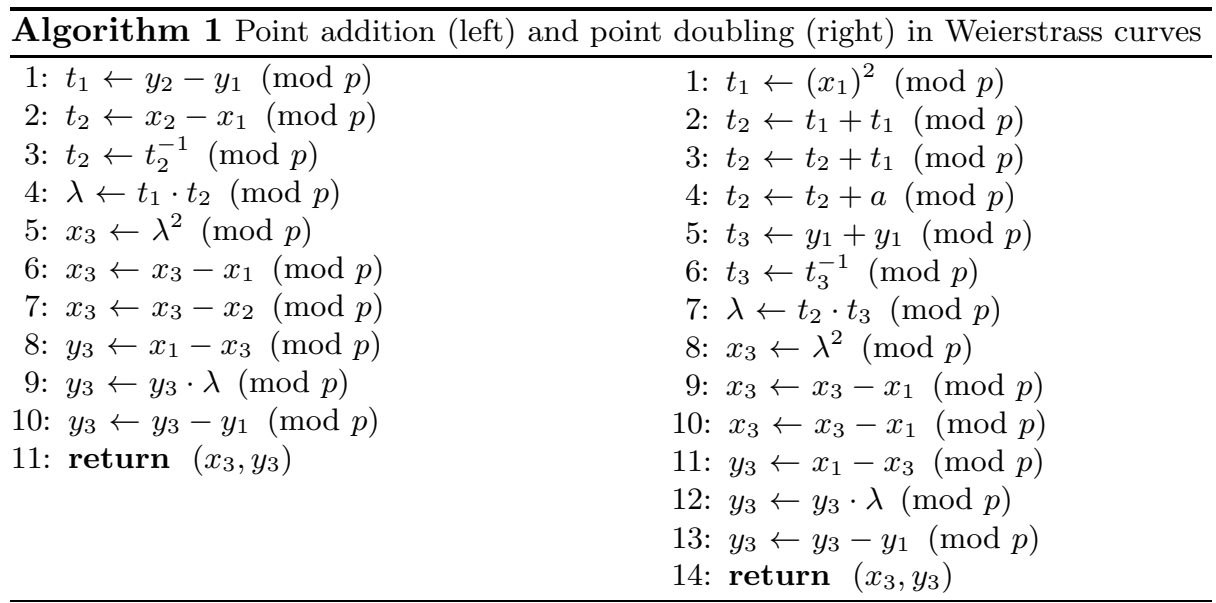


Algorithm 2 shows the details of the point addition in Edwards curves, where no restriction applies to the points $P_{1}=\left(x_{1}, y_{1}\right)$ and $P_{2}=\left(x_{2}, y_{2}\right)$. This implementation requires 4 additions, 9 multiplications, and 2 inversions in $\mathbb{F}_{p}$.

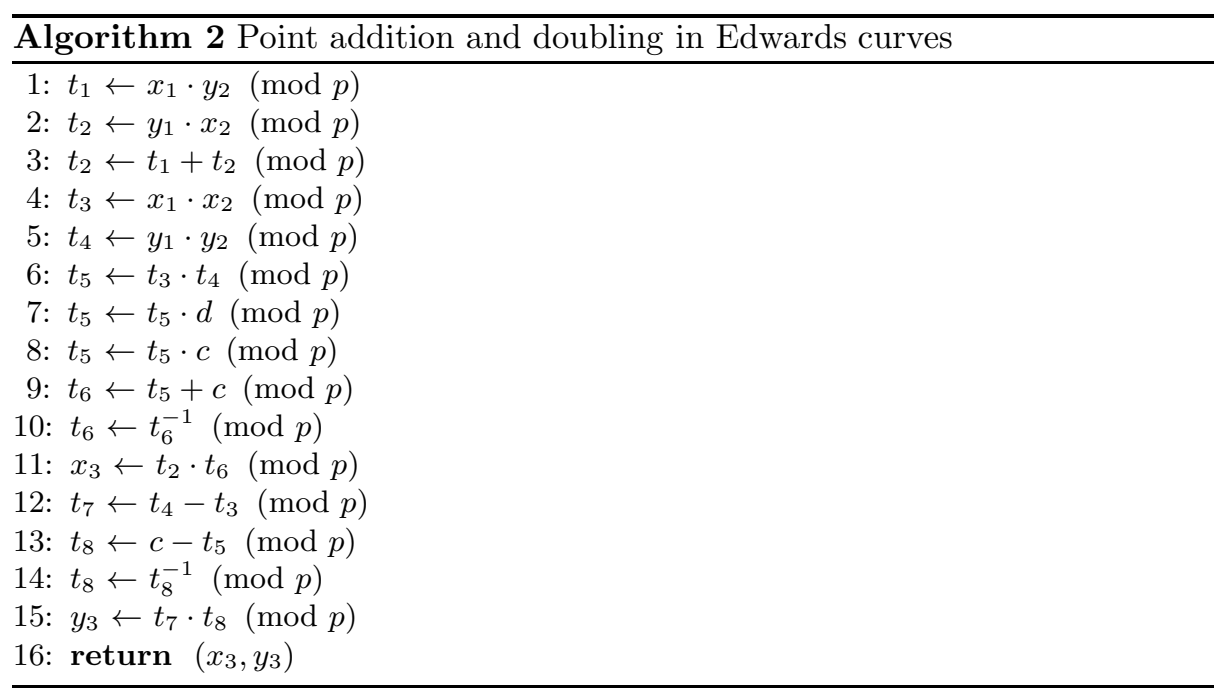

Finally, Algorithm 3 shows the details of the point addition and point doubling operations in Montgomery curves. Similar to Weierstrass curves, in the case of point addition the elements involved are $P_{1}=\left(x_{1}, y_{1}\right)$ and $P_{2}=\left(x_{2}, y_{2}\right)$ such that $P_{1} \neq \pm P_{2}$. This point addition implementation for Montgomery curves needs 10 additions, 9 multiplications, 1 squaring, and 1 inversion in $\mathbb{F}_{p}$, while the point doubling implementation requires 12 additions, 9 multiplications, 2 squarings, and 1 inversion in $\mathbb{F}_{p}$.

Table 1 summarises the operations needed for both the addition and doubling operations with the three types of curves considered in this work.

Table 1: Comparison of the number of operations needed

\begin{tabular}{lcccccccccccc}
\hline & \multicolumn{3}{c}{ Edwards } & \multicolumn{3}{c}{ Montgomery } & \multicolumn{3}{c}{ Weierstrass } \\
& Add. Mul. Sqr. Inv. & Add. Mul. Sqr. Inv. & Add. Mul. Sqr. Inv. \\
\hline Addition & 4 & 9 & 0 & 2 & 10 & 9 & 1 & 1 & 6 & 2 & 1 & 1 \\
Doubling & 4 & 9 & 0 & 2 & 12 & 9 & 2 & 1 & 8 & 2 & 2 & 1 \\
\hline
\end{tabular}




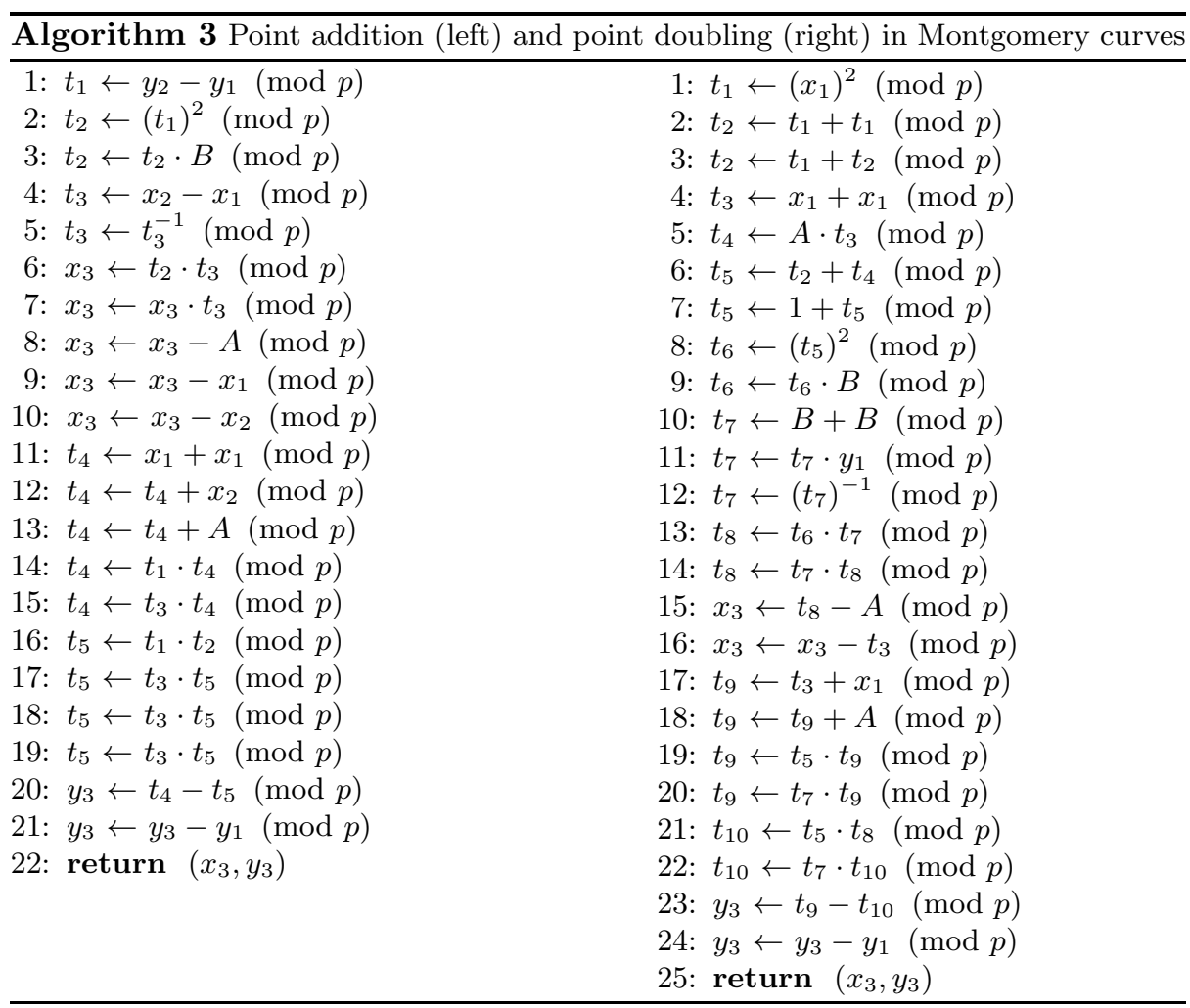

\section{Experimental results}

The tests whose results are shown in this section were performed using an Intel Core i7 processor model 2630QM at $2.00 \mathrm{GHz}$, while the Java application was compiled with the Java Development Kit version 1.8.0_66.

We have used the six Edwards curves displayed in SafeCurves as starting point, using the formulas included in this contribution for obtaining the associate Montgomery and short Weierstrass curves. Those curves are the ones identified as E-222 (222 bits), Curve1174 (251 bits), E-382 (382 bits), Curve41417 (414 bits), Ed448-Goldilocks (448 bits), and E-521 (521 bits). The rationale for choosing specifically those curves is that they can be expressed in the three formats (Edwards, Montgomery, and Weierstrass), which in general is not always possible, as for example Montgomery curves cannot always be represented as Edwards curves.

Starting with each base point offered in SafeCurves for the Edwards curves, we have used that point (and the corresponding points of the Montgomery and Weierstrass curves) inside a loop that computes one million addition operations and another million doubling operations. Table 2 shows the running time in seconds of these tests. 
Table 2: Running time in seconds for $10^{6}$ point addition/doubling operations

\begin{tabular}{lrrrrrr}
\hline & \multicolumn{2}{c}{ Edwards } & \multicolumn{2}{c}{ Montgomery } & \multicolumn{2}{c}{ Weierstrass } \\
& Addition & Doubling & Addition & Doubling & Addition & Doubling \\
\hline E-222 & 81.5329 & 84.2961 & 57.4369 & 59.4419 & 41.6957 & 44.9321 \\
Curve1174 & 102.8423 & 102.8315 & 70.1401 & 72.3692 & 50.6845 & 54.3952 \\
E-382 & 175.2261 & 182.7206 & 129.0495 & 129.0018 & 89.7586 & 96.1143 \\
Curve41417 & 193.9862 & 202.3829 & 142.7497 & 142.2198 & 99.3412 & 106.2448 \\
Ed448 & 216.1019 & 225.5697 & 158.5627 & 156.1755 & 110.7912 & 117.9702 \\
E-521 & 284.5245 & 297.9542 & 212.0656 & 209.4322 & 145.9185 & 155.5230 \\
\hline
\end{tabular}

\section{Conclusions}

As it was expected, given the number and type of operations needed for the addition and doubling operations in each curve, the best results have been obtained with curves of the short Weierstrass type, and the worst results have been produced by the Edwards curves. More specifically, the running time with Edwards curves is almost twice the time needed when using Weierstrass curves, which have the simpler definition for the operations tested.

If we consider negligible the running time for additions, and join the squaring operations with the multiplications, then the relationship obtained between multiplications and inversions is almost 1:100, which means than an inversion is, from the computational point of view, as costly as 100 multiplications. This value matches the estimation of [16], so we have been able to confirm that data in our testing environment.

Across different tests we have always obtained a small but noticeable difference in the results for both operations in Edwards curves. Given that exactly the same algorithm is used in both cases, the difference can only be explained by the specific points used in each test, which are not the same. Nevertheless, that result deserves a more-in-depth investigation in the next phase of our research, together with an extension of the research so it takes into consideration alternative coordinate systems (projective, Jacobian, etc.).

\section{Acknowledgment}

This work has been partially supported by Ministerio de Economía, Industria y Competitividad (MINECO), Agencia Estatal de Investigación (AEI), and Fondo Europeo de Desarrollo Regional (FEDER, UE) under project COPCIS, reference TIN2017-84844-C2-1-R, and by Comunidad de Madrid (Spain) under project CIBERDINE, reference S2013/ICE-3095-CM, also co-financed by European Union FEDER funds. 


\section{References}

1. Koblitz, N.: Elliptic curve cryptosytems. Mathematics of Computation 48(177) (1987) 203-209

2. Miller, V.S.: Use of elliptic curves in cryptography. Lecture Notes in Computer Science 218 (1986) 417-426

3. American National Standards Institute: Public Key Cryptography for the Financial Services Industry: Key Agreement and Key Transport Using Elliptic Curve Cryptography. ANSI X9.63. (2001)

4. IEEE: Standard specifications for public key cryptography. Institute of Electrical and Electronics Engineers, IEEE 1363. (2000)

5. National Institute of Standard and Technology: Digital Signature Standard (DSS). NIST FIPS 186-4. (2009) http://nvlpubs .nist.gov/nistpubs/FIPS/NIST.FIPS. 186-4.pdf.

6. Brainpool: ECC Brainpool standard curves and curve generation. Ver. 1.0. (2005) http://www.ecc-brainpool.org/download/Domain-parameters.pdf.

7. Lochter, M., Merkle, J.: Elliptic Curve Cryptography (ECC) Brainpool standard curves and curve generation. (2010) Request for comments (RFC 5639), Internet Engineering Task Force.

8. Bernstein, D.J., Lange, T.: SafeCurves (2014) http://safecurves.cr.yp.to/.

9. Baignères, T., Delerablée, C., Finiasz, M., Goubin, L., Lepoint, T., Rivain, M.: Trap Me If You Can. Million Dollar Curve. (2016) https://cryptoexperts.github.io/million-dollar-curve/specifications/ 2016-02-01_trap-me-if-you-can.pdf.

10. Durán Díaz, R., Gayoso Martínez, V., Hernández Encinas, L., Martín Muñoz, A.: A study on the performance of secure elliptic curves for cryptographic purposes. In: Proceedings of the International Joint Conference SOCO'16-CISIS'16-ICEUTE'16. (2016) 658-667

11. Menezes, A.J.: Elliptic curve public key cryptosystems. Kluwer Academic Publishers, Boston, MA, USA (1993)

12. Cohen, H., Frey, G.: Handbook of elliptic and hyperelliptic curve cryptography. Discrete Mathematics and its Applications. Chapman \& Hall/CRC, Boca Raton, FL, USA (2006)

13. Bernstein, D.J., Lange, T.: Curve25519: New Diffie-Hellman speed records. In: Public Key Cryptography (PKC 2006), 9th International Conference on Theory and Practice in Public-Key Cryptography. (2007) 207-228

14. National Security Agency: NSA Suite B cryptography. (2009) http://www.nsa. gov/ia/programs/suiteb_cryptography/index.shtml.

15. Bundesamt für Sicherheit in der Informationstechnik: Elliptic curve cryptography. BSI TR-03111 version 2.0. (2012) https://www.bsi.bund.de/SharedDocs/ Downloads/EN/BSI/Publications/TechGuidelines/TR03111/BSI-TR-0311__ pdf .pdf?_-_blob=publicationFile.

16. Bernstein, D.J., Lange, T.: Explicit-formulas database (2016) https:// hyperelliptic.org/EFD/.

17. Edwards, H.M.: A normal form for elliptic curves. Bulletin of the American Mathematical Society 44 (2007) 393-422

18. CryptoExperts SAS: The Million Dollar Curve. (2016) https://cryptoexperts. github.io/million-dollar-curve/.

19. Blum, L., Blum, M., Shub, M.: A simple unpredictable pseudo random number generator. SIAM Journal on Computing 15(2) (1986) 364-383 